\title{
HYPOTHERMIA IN A PATIENT TREATED WITH ANTIPSYCHOTICS
}

\author{
Pavlina A. Andreeva-Gateva, ${ }^{1,2}$, \\ Tzvetelin N. Gatev ${ }^{3}$, \\ Ralitza Nachova ${ }^{2}$, \\ Radka K. Tafradjiiska- \\ Hadjiolova ${ }^{4}$, \\ Maria Grozeva ${ }^{5}$
}

${ }^{1}$ Department of Pharmacology and Toxicology, Medical University of Sofia

${ }^{2}$ Department of Pharmacology, Faculty of Medicine, Sofia University "St. Kliment Ohridsky"

${ }^{3}$ Department of Forensic Medicine, Military Hospital, Sofia

${ }^{4}$ Department of Pathophysiology, Medical University of Sofia

${ }^{5}$ Department of Forensic Medicine, Faculty of Medicine, Sofia University "St. Kliment Ohridsky"

\section{Corresponding author:}

Pavlina Andreeva-Gateva

Department of Pharmacology and

Toxicology

Pre-clinical Study Center

Faculty of Medicine

Medical University Sofia

2, Zdrave str.

Sofia, 1421

Bulgaria

e-mail:pandreeva_gateva@outlook.com

Received: October 09, 2015

Revision received: February 26, 2016

Accepted: May 30, 2016

\section{Summary}

We present a case of 58-year-old female patent with a long-term history of paranoid schizophrenia treated with different antipsychotics. She died with sign and symptoms of hypothermia. Autopsy revealed an ischemic stroke. We could not exclude involvement of antipsychotic medications as culprits.

Kew words: hypothermia, ischemic stroke, schizophrenia, antipsychotic drugs

\section{Introduction}

Schizophrenia is a chronic psychiatric disorder, affecting $1 \%$ of the population worldwide. In Bulgaria, roughly 80000 individuals suffer from schizophrenia, although no more than 35000 of them follow a systemic treatment.

Antipsychotic medicaments need to be taken for a long time, exposing patients to the risk of adverse reactions. Hypothermia is a well-known adverse effect of antipsychotic drugs [1]. Cardiovascular incidents (stroke, embolism) are not at all rare in patients treated for schizophrenia, and can present with lower than normal body temperature $[2,3]$.

Schizophrenia is related with impaired social adaptation, working capability, thinking and emotional reactions. Generally, life expectancy of people with schizophrenia is $12-15$ years shorter than that in those without schizophrenia.

The leading causes of death in individuals with schizophrenia remain cardiovascular incidents, including stroke. Results from a population survey lead to conclusions that cardiovascular risk factors monitoring must be initiated at the moment of diagnosis, especially in women.

Hypothermia in patients on antipsychotic treatment is a serious, unpredictable, type B adverse effect, often leading to hospitalization and sometimes to death [4]. Patients with brain disorders are more prone to hypothermic reactions. The preoptic anterior hypothalamic region regulates body temperature and lesions in this part of the brain can cause hypothermia in cases neuroleptics are applied. Patients with schizophrenia suffer from hypothermia more often than patients taking antipsychotic drugs. Neurotensin is suspected to have a leading role, being a thermoregulatory protein 
found to be of lower levels in patients with schizophrenia [5].

The hypothalamic thermic reaction also depends on the ambient temperature.

Body temperature of $35^{\circ} \mathrm{C}$ could be associated with disturbances of the enzyme system activity, disturbances of thermoregulation and compensatory mechanisms. The signs and symptoms include increasing tiredness, adynamia, somnolence, and coma. The symptoms of and histopathology are consistent with hypothermia due to overcooling during transportation for consultation to a general hospital.

Predisposing factors for hypothermia are decreased reactivity of the organism (as a result of illness, including psychical one), medications applied (i.e. barbiturates, antidepressants, phenothiazines etc.) even in therapeutic dosages, poor nutrition and hypoglycemia, lack of physical activity, inappropriate clothing etc. [6].

\section{Case Report}

A 58-year-old female patient with medical history of progredient type paranoid schizophrenia, dating back to 1973 , was admitted on a state psychiatric hospital because of exacerbation on October 8, 2009. The patient was left her home for several days, and was found to attack both verbally and physically mothers in a public garden pretending she wants protect their children.

Her medication history included haloperidol $10 \mathrm{mg}$, chlorpromazine hydrochloride $50 \mathrm{mg}$, and diazepam $20 \mathrm{mg}$, changed to olanzapine $10 \mathrm{mg}$ intramuscularly before admission.
Inpatient treatment included chlorprothixene $30 \mathrm{mg}$ tid, haloperidol $1.5 \mathrm{mg}$ tid, hydroxyzine 25 $\mathrm{mg}$ tid, and biperiden $2 \mathrm{mg}$ tid. While in hospital, ten weeks later (December 16, 2009, 9 am), the patient developed hypothermia (axillar temperature $35^{\circ} \mathrm{C}$ ). She was difficult to establish contact with, somnolent, with arterial blood pressure (BP) 130/80 $\mathrm{mmHg}$.

The ambient temperature those days was $-1^{\circ} \mathrm{C}$, without rain or snow, and the central heating was on for several hours a day, with additional blankets given to patients.

Warmers and bed-rest were ordered without effect on the general condition and body temperature.

Clinical laboratory investigations revealed leucopenia. The result from X-ray evaluation was unremarkable. Neurological examination did not reveal any neurological deficit. Assessment of the thyroid function was unremarkable too. Cortisol was not evaluated. There were no external signs of injury or infection. An EKG revealed sinus bradycardia and non-specific ST segment changes. At 5.30 p.m. the patient vomited and inhaled gastric content.

Later on the same day she was transferred to a municipal hospital with bradycardia, hypotension and increasing adynamia, somnolence, and disorientation. The patient opened her eyes in response to voices and reacted with withdrawal to painful stimuli (Glasgow Coma Scale 11/15). At 9 p. m. on the same day the patient died.

Autopsy revealed that the cause of death was an ischemic stroke (Figure 1), followed by vomiting and aspiration of gastric content (Figure 2).

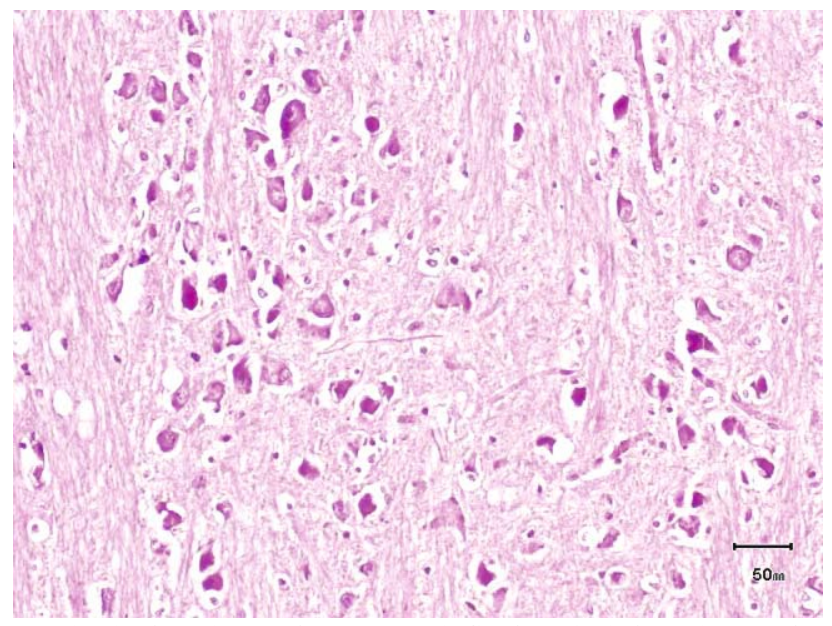

Figure 1. Microscopic diagnosis: ischemic stroke. Hypoxic/ischemic injury in cerebral cortex - "red neuron" shrunken cells, axonal spheroids at points of axonal disruption, swollen cell bodies and peripheral dispersion of 


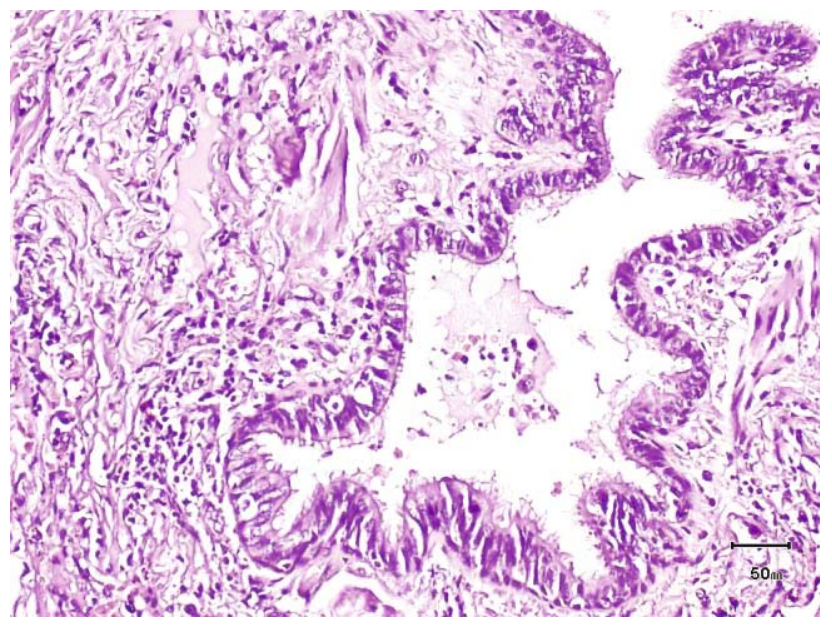

Figure 2. Microscopic diagnosis: aspiration pneumonia. Aspirated material in the bronchi and peribronchial neutrophil infiltration are shown (hematoxylin and eosin stain)

\section{Discussion}

A decrease of the body temperature up to $35^{\circ} \mathrm{C}$ per se would not be necessarily mortal. Prolonged exposure to low environmental temperature is only an additional factor in this case.

In patients with schizophrenia, body temperature is lower due to administration of neuroleptic drugs more often than in patients with other diseases such as delirium or dementia [7]. It is assumed that the main reason for that is neurotensin - a basic thermostatic protein that is lower in patients with schizophrenia [5]. Hypothalamic reaction also depends on the ambient temperature. In certain cases, the overlay of infections acts to further worsen thermoregulation.

In the European database on reported possible side effects of drugs, there are 134 registered cases of hypothermia for olanzapine (the active ingredient in Zyprexa), 54 cases - for haloperidol, and 3 cases - for chlorprothixenum.

Body temperature of $35^{\circ} \mathrm{C}$ (as in the case reported) leads to disturbances in the activity of the enzyme systems and disorder in the thermoregulation, and the compensating capabilities of the body [8]. This occurs with progressively increasing fatigue, adynamia, lethargy, loss of orientation, and somnolence followed by a sleep coma [9].

A drop in body temperature itself within these limits (since there was no documented lower temperatures the patient) would not lead to coma and death. Prolonged exposure to low ambient temperature should not be considered as a leading cause of ensuing death. Rather, the causes are complex.

Psychotropic drugs can lead to the development of metabolic syndrome - its frequency is as high as $67 \%$ in patients with schizophrenia and $49 \%$ in those with bipolar disorder. The risk of development of metabolic syndrome and type 2 diabetes is significantly higher in patients with mental illness than in the general population. The incidence of diabetes in schizophrenic patients reaches $10-15 \%$. Data from epidemiological studies suggests an increased incidence of insulin resistance and diabetes in this group [10]. Hyperglycemia can lead to a coma at very high blood sugar levels (3-6 times above normal). In our case, there was no evidence of abnormal blood glucose.

In each disease, including schizophrenia, the agonal period typically occurs with lowering the body temperature of the dying. The ensuing lower temperature is associated as a complication of the ensuing stroke, aspiration lesions of the lungs and respiratory system and the potent antipsychotic therapy [11]. Based on the data about the state of the patient, the team of forensic examiners concluded that death resulted from the ischemic stroke (as shown on microscopy) which led to vomiting of gastric contents and inhalation thereof, with the development of Mendelson syndrome (chemical pneumonia caused by aspiration of gastric contents into the structures of the lung), as evidenced by the corrosive tracheitis, aspiration pneumonia and bronchiolitis.

The ensuing low temperature was considered 
a complication of the stroke, aspiration lesions of the lungs and respiratory system and the ongoing potent antipsychotic therapy.

\section{References}

1. López-Muñoz F, Alamo C, Cuenca E, Shen WW, Clervoy P, Rubio G. History of the discovery and clinical introduction of chlorpromazine. Ann Clin Psychiatry. 2005;17:113-35.

2. National Center for Biotechnology Information. PubChem Compound Database; $\mathrm{CID}=3559$ [Internet]. Bethesda (MD): U.S. National Library of Medicine; 2001 [cited 2015 Sept 16]. Available from:

https://pubchem.ncbi.nlm.nih.gov/compound/355 9.

3. Sweeting J, Duflou J, Semsarian C. Postmortem analysis of cardiovascular deaths in schizophrenia: a 10-year review. Schizophr Res. 2013;150:398403.

4. Rummel-Kluge C, Komossa K, Schwarz S, Hunger H, Schmid F, Lobos CA, et al. Head-tohead comparisons of metabolic side effects of second generation antipsychotics in the treatment of schizophrenia: a systematic review and metaanalysis. Schizophr Res. 2010;123:225-33.

5. Osadchii OE. Emerging role of neurotensin in regulation of the cardiovascular system. Eur J Pharmacol. 2015;762:184-92.
6. Young DM. Risk factors for hypothermia in psychiatric patients. Ann Clin Psychiatry. 1996;8:93-7.

7. Fusar-Poli P, Smieskova R, Kempton MJ, Ho BC, Andreasen NC, Borgwardt S. Progressive brain changes in schizophrenia related to antipsychotic treatment? A meta-analysis of longitudinal MRI studies. Neurosci Biobehav Rev. 2013;37:168091.

8. Kreuzer $\mathrm{P}$, Landgrebe $\mathrm{M}$, Wittmann $\mathrm{M}$, Schecklmann M, Poeppl TB, Hajak G, et al. Hypothermia associated with antipsychotic drug use: a clinical case series and review of current literature. J Clin Pharmacol. 2012;52:1090-7.

9. Moncrieff J, Cohen D, Mason JP. The subjective experience of taking antipsychotic medication: a content analysis of Internet data. Acta Psychiatr Scand. 2009;120:102-11.

10. Murray-Thomas T, Jones ME, Patel D, Brunner E, Shatapathy CC, Motsko S, et al. Risk of mortality (including sudden cardiac death) and major cardiovascular events in atypical and typical antipsychotic users: a study with the general practice research database. Cardiovasc Psychiatry Neurol. 2013;2013:247486.

11. van Marum RJ, Wegewijs MA, Loonen AJ, Beers E. Hypothermia following antipsychotic drug use. Eur J Clin Pharmacol. 2007;63:627-31. 